\title{
Langmuir Probe Measurements of Scrape-Off Layer Conditions in RF-Driven Plasmas in TST-2*)
}

\author{
James H.P. RICE, Naoto TSUJII, Yuichi TAKASE, Akira EJIRI, Osamu WATANABE, Hibiki \\ YAMAZAKI, Yi PENG, Kotaro IWASAKI, Yuki AOI, Yongtae KO, Kyohei MATSUZAKI \\ and Yuki OSAWA
}

The University of Tokyo, Kashiwa 277-8561, Japan

(Received 28 November 2019 / Accepted 20 February 2020)

\begin{abstract}
A new Langmuir probe has been designed and installed in TST-2 for measurements of Scrape-Off Layer plasmas (SOL). Non-inductive current drive is considered essential for spherical tokamak reactors. It has previously been shown that a large amount of injected Lower Hybrid Wave (LHW) power is lost in the SOL [1]. A full density profile of SOL conditions is necessary to accurately simulate the propagation of LHW in TST-2. A new probe was designed for durability, larger signal and Mach probe measurements. The new probe has been installed in TST-2 and results have been obtained. Temperature measurements show $T_{\mathrm{e}}=30-50 \mathrm{eV}$ during RF injection and $<10 \mathrm{eV}$ otherwise. Density measurements show $n_{\mathrm{e}}=2.0 \times 10^{15} \mathrm{~m}^{-3}$ and $1.5 \times 10^{16} \mathrm{~m}^{-3}$ during flat-top RF power injection from Outboard- and Top-launch antennas, respectively. This is above the cut-off density for the $200 \mathrm{MHz}$ LHW $\left(5 \times 10^{14} \mathrm{~m}^{-3}\right)$ in TST-2, thus LHW can propagate through SOL plasma.

(c) 2020 The Japan Society of Plasma Science and Nuclear Fusion Research
\end{abstract}

Keywords: Langmuir probe, Mach probe, lower-hybrid wave, spherical tokamak

DOI: $10.1585 /$ pfr. 15.2402009

\section{Introduction}

Lower-Hybrid Current Drive (LHCD) is potentially a highly efficient method for current drive in spherical tokamak fusion reactors. However, LHCD suffers from limitations at high density where current drive efficiency is seen to decrease. Experiments on many devices [2-4] have indicated lower efficiency than predictions of numerical simulations [5].

One possible cause is thought to be LH power losses in SOL plasma. Previous research has identified a number of processes that cause LH power loss, such as Landau damping [1], collisional processes [6], parametric decay instabilities [2], or SOL density fluctuations [7]. To investigate the effect these processes have on TST-2's LHCD scenario, a full profile of SOL plasma in the TST- 2 spherical tokamak is desired.

Due to the lower temperature and density of SOL plasma, electrostatic Langmuir probes [8] make an appropriate solution. Langmuir probes simultaneously provide measurements of plasma density, temperature and floating potential $\left(n_{\mathrm{e}}, T_{\mathrm{e}}\right.$, and $\left.V_{\mathrm{fl}}\right)$ by sweeping the bias voltage. These parameters can be obtained by fitting the measured characteristic I-V curve following the relationship:

$$
I_{\text {probe }}=I_{\text {sat }}\left(1-\exp \frac{e\left(V-V_{\mathrm{fl}}\right)}{T_{\mathrm{e}}}\right),
$$

where $T_{\mathrm{e}}$ is the electron temperature, $V_{\mathrm{fl}}$ is the plasma

author'se-mail: rice@fusion.k.u-tokyo.ac.jp

*) This article is based on the presentation at the 28th International Toki Conference on Plasma and Fusion Research (ITC28). floating potential, and $I_{\text {sat }}$ is the ion saturation current:

$$
I_{\mathrm{sat}}=-n_{\mathrm{i}} e Z S_{\mathrm{p}} \exp \left(-\frac{1}{2}\right) \sqrt{\frac{T_{\mathrm{e}}}{m_{\mathrm{i}}}},
$$

dependant on the probe area $S_{\mathrm{p}}$, charge $Z_{\mathrm{e}}$, ion mass $m_{\mathrm{i}}$, and the ion density $n_{\mathrm{i}}$.

Previous Langmuir probes used in TST-2 were installed on the antenna limiters. The probes saw large fluctuations in the probe current during RF pulse, and it was difficult to obtain the I-V curves reliably. The new probes were installed away from the LH antennae to avoid strong local perturbations by the $\mathrm{LH}$ antennae.

In this work, a newly designed probe was tested. We aimed to improve the accuracy of density measurements in TST-2's SOL plasma, and to identify issues preventing reliable temperature measurements. These are important steps towards implementing a full and accurate plasma profile of TST-2.

\section{Experimental Set-Up}

Experiments were performed on the TST-2 spherical tokamak [9] at The University of Tokyo. It features design parameters of (major radius $R_{0}=0.36 \mathrm{~m}$, minor radius $a=0.23 \mathrm{~m}$, aspect ratio $A>1.6$, toroidal magnetic field $B_{\mathrm{t} 0}<0.3$ ), and can produce $I_{\mathrm{p}}<27 \mathrm{kA}$ during noninductive current drive. This is achieved using its $200 \mathrm{MHz}$ LHCD system. This consists of Top- and Outboard-launch antennae, currently driven by three $100 \mathrm{~kW}$ amplifiers allowing for a maximum of $200 \mathrm{~kW}$ of Outboard-launch 


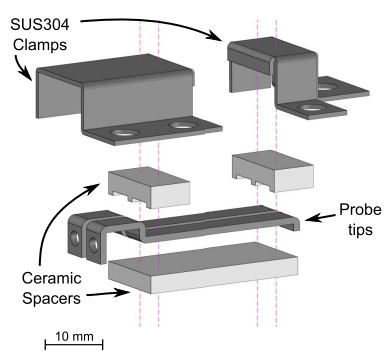

(a)

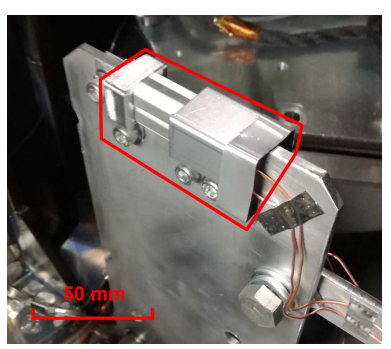

(b)
Fig. 1 (a) Exploded view of the probe, showing assembly of the probe clamping method, and (b) the probe installed on the lower limiter.

power and $100 \mathrm{~kW}$ of Top-launch power.

Langmuir probe measurements were taken using a newly designed configuration tested in $\mathrm{D}_{2}$ plasma. Figure 1 shows an exploded view of one side of the probe configuration and the probe after initial installation. The new design features four channels, two on either side of the limiter, using $20 \times 6 \mathrm{~mm}$ probe tips located $5 \mathrm{~mm}$ and $13 \mathrm{~mm}$ from the plasma limiter edge. Results presented in this study were obtained using the upper $(5 \mathrm{~mm})$ probes. The probes are made from SUS304 and are attached to the limiter with clamps and are electrically isolated using ceramic spacers. This clamping method avoids pinpoint stresses on ceramic parts and improves the durability of the probe. The new probe design has yet to fail after 9 months of continuous operation.

Additionally, previous probes [10] had probe areas of $20-40 \mathrm{~mm}^{2}$ and drew $I_{\text {sat }}<1 \mathrm{~mA}$, which was insufficient to overcome noise, especially during the RF pulse. In the new design, probe area was increased by 3-6 times, to measure lower-density SOL plasma, giving $5-10 \mathrm{~mA}$. This has greatly improved the quality of the probe signal.

This probe is located at the lower extremity of the plasma, at $(R, Z)$ coordinates $(+275,-450) \mathrm{mm}$ and toroidal location $\phi=-60^{\circ}$ as seen in Fig. 2. The probe bias voltage was swept with a sinusoidal wave with frequency range of $1-40 \mathrm{kHz}$, and the voltage range was adjusted to $(-165 \mathrm{~V}-+15 \mathrm{~V})$ to give a current range of about $\pm I_{\mathrm{sat}}$. This helped to ensure the detection circuit (i.e., the pick-up resistor) did not become overloaded by the now increased probe current. The current signal was usually detected using a $470 \Omega$ resistor, but $0.1,1.0$ and $4.7 \mathrm{k} \Omega$ resistors are also available. The I-V curves were then fitted to obtain the density, temperature and potential. Plasma current convention defines the downstream side as open-facing to electron flow, while upstream is facing away.

\section{Results}

\subsection{Typical Scrape-Off Layer conditions}

Results were obtained using the new Langmuir probe during non-inductive operation using LHW power from the Top- or Outboard-launch antennae. A representative time

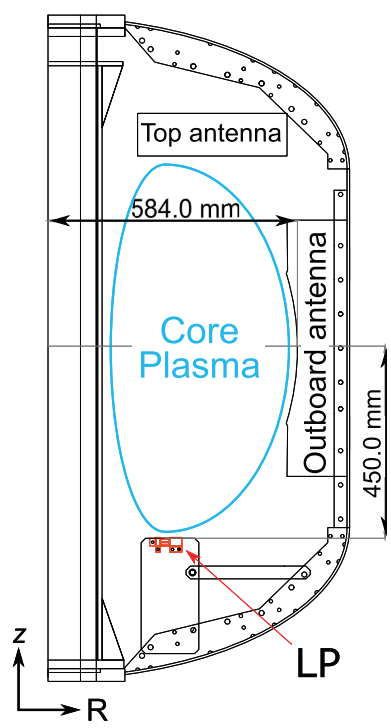

(a)

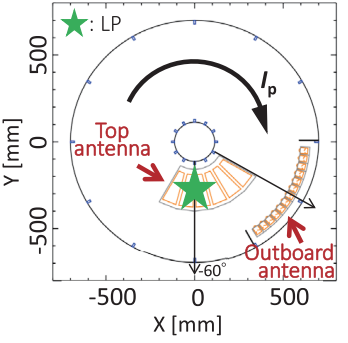

(b)
Fig. 2 (a) Poloidal and (b) toroidal cross sections (top view) of TST-2 showing location of limiters, LHW antennae and the new Langmuir probes located at $(R, Z)=(+275,-450)$ and $\phi=-60^{\circ}$.

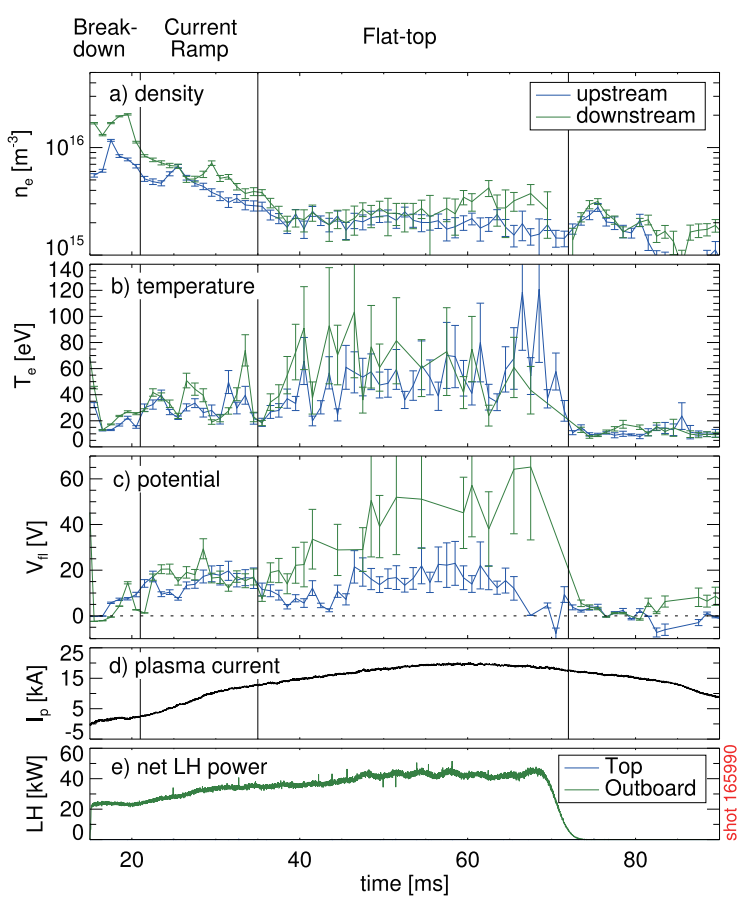

Fig. 3 Representative time traces of (a) density $n_{\mathrm{e}}$, (b) electron temperature $T_{\mathrm{e}}$, c) floating potential $V_{\mathrm{ff}}$, d) plasma current, $I_{\mathrm{p}}$, and e) net LH power taken during an Outboardlaunch LHW discharge.

trace of plasma parameters during each launch configuration are shown in Figs. 3 and 4.

Typical results show density during flat-top LHW power to be $n_{\mathrm{e}}=2.0 \times 10^{15} \mathrm{~m}^{-3}$ and $1.5 \times 10^{16} \mathrm{~m}^{-3}$ for Outboard- and Top-launch experiments, respectively. These results are consistent with previous results [10] 


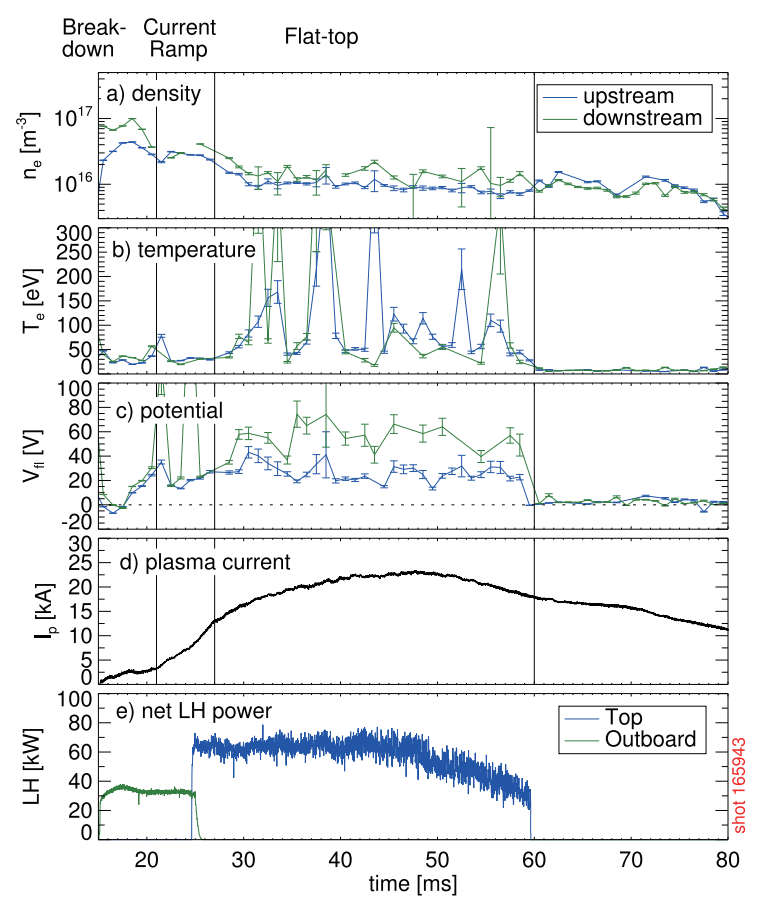

Fig. 4 Representative time traces of a) density, $n_{\mathrm{e}}$, b) electron temperature, $T_{\mathrm{e}}$, c) floating potential $V_{\mathrm{fl}}$, d) plasma current, $I_{\mathrm{p}}$, and e) net $\mathrm{LH}$ power taken during a Top-launch LHW discharge.

which showed TST-2's SOL density to be roughly $2.0 \times 10^{15} \mathrm{~m}^{-3}$ during Outboard-launch.

Typical temperatures of $T_{\mathrm{e}} \sim 30-100 \mathrm{eV}$ were found during Top- and Outboard-launch LHW power injection and $<10 \mathrm{eV}$ while there is no injected power. It is suspected this is an overestimate due to the influence of RF power and is discussed in Sec 3.3. These results show that the SOL density is above the cut-off density of the $200 \mathrm{MHz}$ LHW $\left(5 \times 10^{14} \mathrm{~m}^{-3}\right)$ up to the limiter.

\subsection{Flow measurements}

Additional information can be obtained using a Mach Probe-like analysis [8] of Langmuir probes installed on the upstream and downstream side of the limiter. The flow of plasma can be observed as a difference in density on either side of the limiter. Assuming approximately equal temperature on either side of the limiter, Mach number of plasma flow can be obtained by:

$$
M_{\infty}=0.45 \ln \left(\frac{I_{\text {sat,down }}}{I_{\text {sat,up }}}\right),
$$

where $M_{\infty}=\frac{v_{\|}}{C_{\mathrm{s}}}$ is the Mach number of the parallel flow across of the limiter and $C_{\mathrm{s}}$ is the ion sound speed. Figure 5 shows the measured toroidal flow using a $40 \mathrm{kHz}$ sweep frequency.

Preliminary results show that, in general, flow is positive $(\sim 0.3)$ during plasma breakdown, near zero during current ramp-up, before returning to positive flow during flat-top power. Curiously, a brief period of reverse flow is

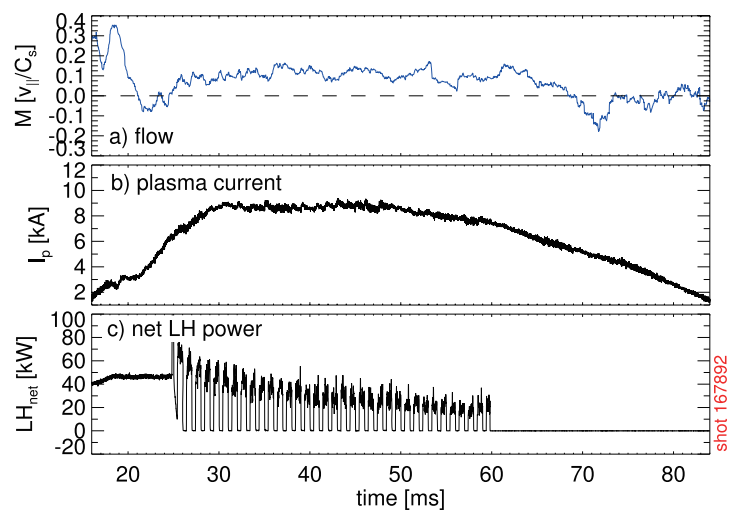

Fig. 5 Flow measurements obtained through flow analysis of Langmuir probe measurements taken at $40 \mathrm{kHz}$ bias voltage sweep frequency, depicting a) Mach number, b) $I_{\mathrm{p}}$, and c) net LH power.

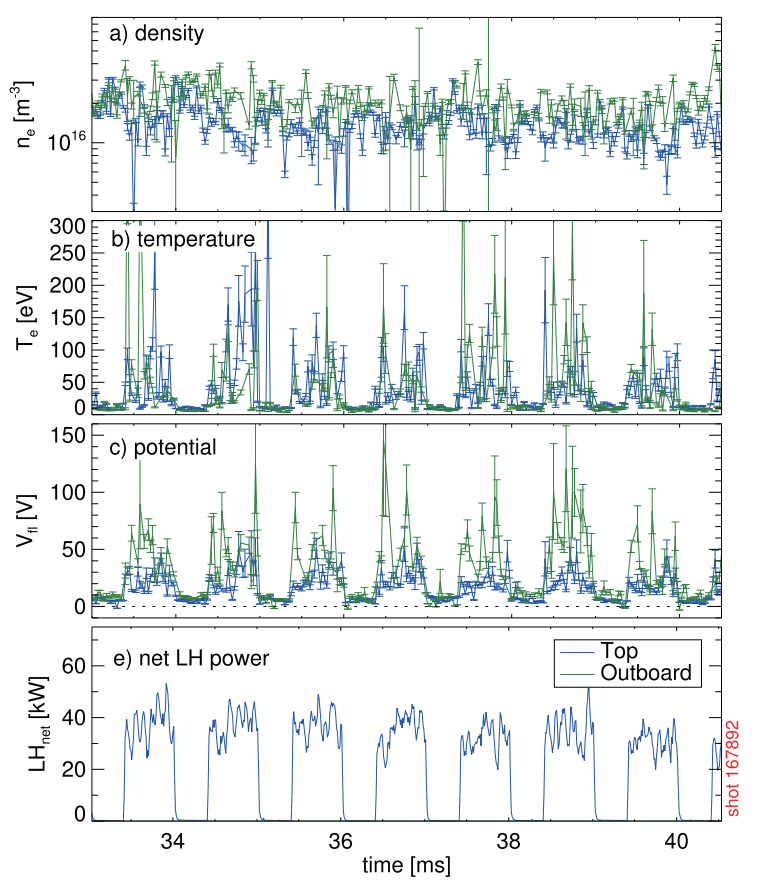

Fig. 6 Time traces of a) density $n_{\mathrm{e}}$, b) electron temperature $T_{\mathrm{e}}, \mathrm{c}$ ) floating potential $V_{\mathrm{fl}}$, and d) net $\mathrm{LH}$ power during power modulation, showing the influence of RF power on Langmuir probe measurements.

regularly observed sometime after LHW power ends. It is not known why this brief reverse flow is seen, and further investigation is needed to clarify this phenomenon.

\subsection{Influence of RF power}

A power modulation experiment was performed (Fig. 6) using the Top-launch antenna at $1 \mathrm{kHz}$ modulation.

The SOL $T_{\mathrm{e}}$ and $V_{\mathrm{fl}}$ signals both respond to RF power. Measurements during on-phase show $T_{\mathrm{e}}$ being similar to LHW-heated plasma, while off-phase $T_{\mathrm{e}}$ is similar to bulk edge $T_{\mathrm{e}}$ measured by Thomson Scattering. Additionally, $V_{\mathrm{fl}}$ is seen to increase during RF power, as seen in Fig. 7. 


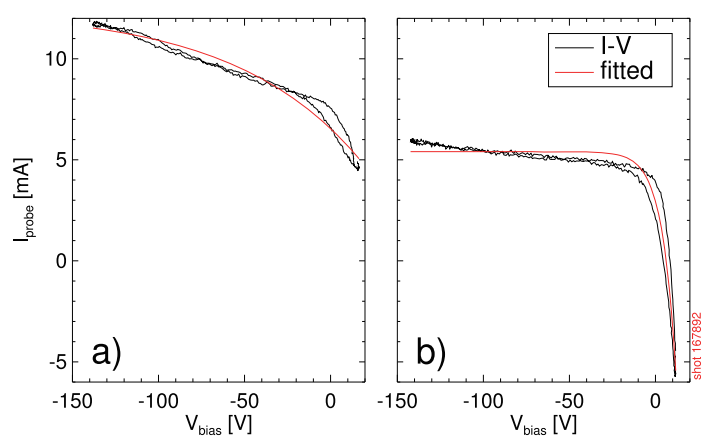

Fig. 7 Ensemble averaged I-V curves taken at a) $30.4 \mathrm{~ms}$ with RF power and b) $31.0 \mathrm{~ms}$ without RF power. Comparison shows the clear increase to $V_{\mathrm{fl}}$ during $\mathrm{RF}$ power.

RF sheath rectification [11] produces a negative $\Delta V_{\mathrm{fl}}$, yet the results presented here show a strongly positive $\Delta V_{\mathrm{fl}}$, indicating that RF sheath rectification is not the dominant alteration to $V_{\mathrm{fl}}$ measurements. The increased loss of fast electrons generated by RF power is thought to increase plasma potential leading to the increase in $V_{\mathrm{fl}}$ seen here.

The increase in $T_{\mathrm{e}}$ could also be due to the generation of fast electrons by RF power. It has long been known that the presence of non-thermal electrons can dominate the Langmuir probe temperature diagnostic [12]. If a thermal Maxwellian electron distribution model is assumed, even a small amount (2\%) of fast electrons can mask bulk $T_{\mathrm{e}}$ signals, leading to erroneously high measurements.

Fast electrons in SOL plasma could be caused by either transport from far off-axis LHCD or by direct heating of SOL plasma by LH power injection. Magnetic equilibrium fitting showed that the downstream probe is magnetically connected to the outboard antennae, but shielded from the top antenna by the inboard limiters. Since alteration is seen in both launch configurations, it is likely caused by fast electron transport.

However, the analysis method used assumed the standard Maxwellian model of electron temperature, so it is not clear whether the observed change in $T_{\mathrm{e}}$ during the RF pulse is due to the presence of fast electrons or a transient increase in the bulk temperature. Calculation of the Electron Energy Distribution Function (EEDF) directly from the I-V probe characteristic [13] may prove critical in future analysis, as it would separate the bulk $T_{\mathrm{e}}$ from fast electron influence, allowing for comprehensive measurements of SOL temperature.

Despite strong alteration to both $T_{\mathrm{e}}$ and $V_{\mathrm{fl}}$, the $n_{\mathrm{e}}$ signal does not respond to the injected RF power.

\section{Summary}

Measurements of plasma parameters in TST-2's SOL region was performed using the newly installed Langmuir probe demonstrated improved signal quality of
SOL density measurements over previous designs. This was achieved by using a probe area roughly 4-6 times larger than previous designs. The downstream and upstream probes show typical densities of $0.3-1.6 \times 10^{16}$ and $0.1-1.2 \times 10^{16} \mathrm{~m}^{-3}$, respectively, during $\mathrm{LH}$ experiments. These results are consistent with previous Langmuir probe measurements of TST-2's SOL density (Top: $\sim 10^{16} \mathrm{~m}^{-3}$, Outboard: $\sim 10^{15} \mathrm{~m}^{-3}$ ). These measurements show the SOL density is above the cut-off density for the $200 \mathrm{MHz}$ LHW.

Results from power modulation experiments show significant alterations to $V_{\mathrm{fl}}$ and $T_{\mathrm{e}}$ during RF operation. A positive change to $V_{\mathrm{fl}}$ is seen, which indicates an enhanced fast electron component being the dominant change to $V_{\mathrm{fl}}$ rather than RF sheath rectification. It is also believed that an enhanced fast electron presence in SOL plasma may have given an erroneously high measurements of $T_{\mathrm{e}}$. An evaluation of the EEDF is necessary to confirm the presence of fast electrons in SOL plasma and determine their effect on measured plasma parameters.

Additionally, the probes were installed on the upstream and downstream sides of the limiter, which allowed for Mach probe analysis.

Future work hopes to confirm the presence of fast electrons and determine their source through EEDF analysis, allowing for more accurate measurements of $T_{\mathrm{e}}$ in the future.

\section{Acknowledgements}

This work was supported by Japan Society for the Promotion of Science Grant-in-Aid for Scientific Research (S) (21226021), by National Institute for Fusion Science Collaboration Research Programs NIFS14KOCR001, NIFS18KOAR022 and NIFS12KUTR078. This work was also supported in part by Japan/US Cooperation in Fusion Research and Development and US DoE Cooperative Agreement DE-FC02-04ER54698.

[1] N. Tsujii et al., Nucl. Fusion 57, 126032 (2017).

[2] Y. Takase et al., Phys. Fluids 28, 983 (1985).

[3] R. Cesario et al., Nat. Commun. 1, 55 (2010).

[4] B.J. Ding et al., Nucl. Fusion 55, 093030 (2015).

[5] N.J. Fisch and A.H. Boozer, Phys. Rev. Lett. 45, 720-2 (1980).

[6] G.M. Wallace et al., Nucl. Fusion 51, 083032 (2011).

[7] E.H. Martin et al., Nucl. Fusion 59, 076006 (2019).

[8] I.H. Hutchinson, Plasma Diagnostics (Cambridge Univ. Press 2005).

[9] Y. Takase et al., Nucl. Fusion 41, 1543 (2001).

[10] T. Shinya et al., Nucl. Fusion 57, 036006 (2017).

[11] R.J. Perkins et al., Nucl. Mater. Energy 12, 283 (2017).

[12] P.C. Stangeby, Plasma Phys. Control. Fusion 37, 1031 (1995).

[13] Tsv.K. Popov et al., Plasma Sources Sci. Technol. 25, 033001 (2016). 